\title{
MEASURE-THEORETIC UNIFORMITY
}

\author{
BY GERALD E. SACKS ${ }^{1}$ \\ Communicated by D. Scott, August 2, 1966
}

Here we present the principal ideas and results of [5] with some indications of proof. We introduce the notion of measure-theoretic uniformity, and we describe its use in recursion theory, hyperarithmetic analysis, and set theory. In recursion theory we show that the set of all sets $T$ such that the ordinals recursive in $T$ are the recursive ordinals has measure 1 . In set theory we obtain all of Cohen's independence results [1], [2] without any use, overt or concealed, of his method of forcing or his notion of genericity. Solovay [8], [9] has extended Cohen's method by forcing statements with closed, measurable sets of conditions rather than finite sets of conditions; in this manner he exploits forcing and genericity to prove: if $Z F$ is consistent, then $Z F+$ "there exists a translation-invariant, countably additive extension of Lebesgue measure defined on all sets of reals" + "the countable axiom of choice" is consistent. Solovay's result is also a consequence of the notion of measure-theoretic uniformity.

We begin with the simplest possible example of measure-theoretic uniformity. Let $T$ be an arbitrary set of natural numbers, and let $P$ be the power set of the natural numbers. We think of $P$ as the product of countably many copies of a two-point set $\{a, b\}$. We assign the unbiased measure: $m(\{a, b\})=1, m(\{a\})=m(\{b\})=\frac{1}{2}$, and $m(\phi)=0$. We give $P$ the induced product measure denoted by $u$.

Let $R(T, x, y)$ be a recursive predicate of the set-variable $T$ and the number variables $x$ and $y$. A familiar uniformity can be expressed as follows: If for some given $T$ we have $(x)(E y) R(T, x, y)$, then there exists a function $f$ recursive in the given $T$ such that $(x) R(T, x, f(x))$. Before we introduce the measure-theoretic counterpart of this uniformity, we must shift our point of view from Skölem functions to bounding functions in order to make the measure come out right: if for some given $T$ we have $(x)(E y) R(T, x, y)$, then there exists a function $f$ recursive in $T$ such that $(x)(E y)_{y \leq f(x)} R(T, x, y)$. Note that the existence of a Skölem function is equivalent to the existence of a bounding function. It is not hard to verify: if $\{T \mid(x)(E y) R(T, x, y)\}$ has measure 1, then $\left\{T \mid(E f)\left(f\right.\right.$ recursive and $\left.\left.(x)(E y)_{y \leq f(x)} R(T, x, y)\right)\right\}$ has measure 1 . Thus the restriction of the bounding function $f$ to the

1 The preparation of this paper was supported by U. S. Army Contract ARO-D. 373. The author wishes to thank Professor Anil Nerode for many helpful conversations on uniformity and definability in set theory. 
recursive functions resulted merely in a restriction of $T$ to a set of measure 1.

Theorem 1. Let $B(T, x, y)$ be arithmetical. Then the set of all $T$ satisfying the following condition has measure $1:$ if $(x)(E y) B(T, x, y)$, then there exists an arithmetical function $f$ such that $(x)(E y)_{y \leq f(x)} B(T, x, y)$.

Corollary 2. Let $B(T)$ be arithmetical. If the set $\hat{T} B(T)$ has positive measure, then $B(A)$ holds for some arithmetical $A$.

The proof of Theorem 1 turns on the fact that the measure of the set $\hat{T} B(T, x, y)$ is an arithmetical function of $x$ and $y$.

Let $T_{0}, T_{1}, T_{2}, \cdots$ be an arbitrary sequence of sets of natural numbers. For all results below prior to Theorem 7 , we define $\mathfrak{N}\left(T_{0}, T_{1}, T_{2}, \ldots\right)$ as follows: let $\Re_{0}\left(T_{0}, T_{1}, T_{2}, \cdots\right)$ be the set of all sets arithmetical in some finite subsequence of $T_{0}, T_{1}, T_{2}, \ldots$; for each recursive ordinal $\alpha>0$, let $\mathfrak{N}_{\alpha}\left(T_{0}, T_{1}, T_{2}, \cdots\right)$ be the set of all sets "hyperarithmetic" in some finite subsequence of $T_{0}, T_{1}, T_{2}, \ldots$ with the set-quantifiers of the hyperarithmetic definitions restricted to $U\left\{\mathscr{T}_{\beta}\left(T_{0}, T_{1}, T_{2}, \cdots\right) \mid \beta<\alpha\right\}$; finally, let $\mathscr{M}\left(T_{0,}, T_{1}, T_{2}, \cdots\right)$ $=\mathrm{U}\left\{\mathrm{TT}_{\alpha}\left(T_{0}, T_{1}, T_{2}, \cdots\right) \mid \alpha<\omega_{1}\right\}$, where $\omega_{1}$ is the least nonrecursive ordinal, We put a probability measure on sequences of sets of natural numbers by putting the product measure on the product of countably many copies of $P$, the power set of the natural numbers. Feferman [3] proves that if $T_{0}, T_{1}, T_{2}, \cdots$ is a generic sequence, then $\mathfrak{m}\left(T_{0}, T_{1}, T_{2}, \cdots\right)$ is a model of the $\sum_{1}^{1}$-axiom of choice. The set of all generic sequences has measure 0 .

TheOREM 3. With probability 1: $\operatorname{Tr}\left(T_{0}, T_{1}, T_{2}, \cdots\right)$ is a model of the $\sum_{1}^{1}$-axiom of choice.

Spector [10] proved the existence of two incomparable hyperdegrees by observing that the set $\left\{\left(T_{0}, T_{1}\right) \mid T_{0}\right.$ and $T_{1}$ are hyperarithmetically incomparable $\}$ has measure 1 . Theorem 3 is a consequence of the measure-theoretic uniformity expressed by Theorem 4. The origin of Theorem 4 is a result of Kreisel [4]: if $B(T, x, y)$ is $\pi_{1}^{1}$, and if for some given $T$ we have $(x)(E y) B(T, x, y)$, then there exists a function $f$ hyperarithmetic in the given $T$ such that $(x) B(T, x, f(x))$.

TheOREM 4. Let $B(T, x, y)$ be $\pi_{1}^{1}$. The set of all $T$ satisfying the following condition has measure 1 : if $(x)(E y) B(T, x, y)$, then there exists a hyperarithmetic function $f$ such that $(x)(E y)_{\nu \leq f(x)} B(T, x, y)$.

CoROLlary 5. With probability 1 , the ordinals recursive in $T$ are the recursive ordinals. 
Corollary 6. For every $T$, if the set $\{X \mid T$ is hyperarithmetic in $X\}$ has positive measure, then $T$ is hyperarithmetic.

Now let $\mathfrak{T}$ be a countable, initial segment of the constructible sets which is a model of $Z F$. For each $T$, let $\mathfrak{T}(T)$ be the set of all sets constructible from $T$ by means of the ordinals of $\mathfrak{T}$. Let $\mathfrak{M T}_{0}(T)$ $=\{T\}$; for each $\alpha>0$ in $\mathfrak{N}$, let $\mathfrak{M}_{\alpha}(T)$ be the set of all subsets of $\mathrm{U}\left\{\mathrm{TT}_{\beta}(T) \mid \beta<\alpha\right\}$ definable by means of some first-order formula of $Z F$ with constants in, and quantifiers restricted to, $U\left\{\mathscr{T}_{\beta}(T) \mid \beta<\alpha\right\}$. Then $\mathfrak{T}(T)=U\left\{\mathfrak{T T}_{\beta}(T) \mid \beta \in \mathscr{M}\right\}$. Feferman [3] provides a language $\mathfrak{L}^{*}$ suitable for dissecting $\mathfrak{T}(T)$. $\mathfrak{L}^{*}$ includes the usual logical symbols, the $\in$-symbol for membership, numerals for all the ordinals in IT, a 3 -symbol to denote the set $T$, abstraction symbols, and restricted quantifier symbols $(E x)_{x<\alpha}$ and $(x)_{x<\alpha}$.

Let $f$ be a function from the ordinals of $m$ into the ordinals of $M$. We say $f$ is definable in $\mathscr{T} t$ if there exists a formula $F(x, y)$ of $Z F$ with constants in $\Re$ such that for all $\alpha, \beta \in \mathscr{N}$, we have $f(\alpha)$ $=\beta \leftrightarrow F_{\mathfrak{N}} F(\alpha, \beta)$. Theorem 7 expresses the key measure-theoretic uniformity we associate with $Z F$.

THEOREM 7. Let $B(x, y)$ be a formula of $\mathfrak{S}^{*}$. Then the set of all $T$ satisfying the following condition has measure $1: i f \vDash \mathscr{T l}(T)(x)(E y) B(x, y)$, then there exists a function $f$ definable in 91 such that

$$
F_{\mathscr{T}^{(T)}}(x)_{x<\alpha}(E y)_{y<i(\alpha)} B(x, y) .
$$

For each sentence $\mathcal{F}$ of $\mathscr{L}^{*}$, we observe that the set $\left\{T \mid \vDash_{\mathfrak{T}(T)} \mathfrak{F}\right\}$ is Borel, since $\mathscr{T}$ is countable; we define $p(\mathcal{F})$, the probability that $F$ is true in $\mathscr{T}(T)$, to be the measure of this Borel set.

THEOREM 8. For each $k \geqq 0$, the function $p(\mathfrak{F})$, restricted to sentences of $\mathfrak{L}^{*}$ having at most $k$ unbounded quantifiers, is definable in $\mathfrak{T}$.

Theorem 7 follows from Theorem 8 easily. Let $p(\alpha, \beta)$ $=p\left((x)_{x<\alpha}(E y)_{y<\beta} B(x, y)\right)$. By Theorem $7, p(\alpha, \beta)$ is definable in $\Re$. For each $\alpha, p(\alpha, \beta)$ is a nondecreasing, bounded function of $\beta$. But then for each $\alpha$, there is a $\gamma$ such that $p(\alpha, \gamma)=$ least upper bound of $\{p(\alpha, \beta) \mid \beta \in \mathscr{N}\}=p\left((x)_{x<\alpha}(E y) \beta(x, y)\right)$; let the least such $\gamma$ be $f(\alpha)$.

It follows from Theorem 7 that the replacement axiom holds in $\operatorname{m}(T)$ with probability 1 . Both Cohen [1], [2] and Solovay [8], [9] show the replacement axiom holds in their models by observing that the forcing relation, restricted to sentences with at most $k$ unbounded quantifiers $(k \geqq 0)$, is definable in $\mathfrak{M}$. We use Theorem 8 instead.

Theorem 9. With probability one, $\operatorname{T(T}(T)$ is a model of $Z F+V \neq L$ and the cardinals of $\mathrm{TH}(T)$ are the same as those of $\mathrm{TH}$. 
Let $T_{0}, T_{1}, T_{2}, \ldots$ be an arbitrary sequence of sets of natural numbers. $\Re\left(T_{0}, T_{1}, T_{2}, \cdots\right)$ is defined like $\Re(T)$, save for some slight complications designed to maximize the symmetry of $\mathfrak{M}\left(T_{0}, T_{1}, T_{2}, \cdots\right)$. Let $\aleph^{*}$ be augmented by symbols $\jmath_{0}, \Im_{1}, J_{2}, \cdots$; Theorems 7 and 8 remain true. Feferman [3] shows that if $T_{0}, T_{1}$, $T_{2}, \ldots$ is a generic sequence, then $\mathfrak{T}\left(T_{0}, T_{1}, T_{2}, \ldots\right)$ is a model of $Z F$ in which the Boolean algebra of all sets of natural numbers has no nonprincipal, maximal ideals.

THEOREM 10. With probability $1, \mathfrak{M}\left(T_{0}, T_{1}, T_{2}, \cdots\right)$ is a model of $Z F$ in which the Boolean algebra of all sets of natural numbers has no nonprincipal, maximal ideals.

The proof of Theorem 10 uses only one trick not used to prove Theorem 9. We formulate this trick as Lemma 11. Let $\tau$ be an arithmetical function from $\omega^{2}$ into $\{0,1\} ; \tau$ induces a transformation of the sentences of $\mathcal{L}^{*}$ as follows: " $n \in J_{j}$ " is replaced by " $n \in J_{j}$ and $\tau(n, j)=0) \bigvee\left(n \in J_{j}\right.$ and $\left.\tau(n, j)=1\right)$." In a similar fashion, $\tau$ transforms all sequences of sets of natural numbers.

Lemma 11. $\quad\left\{\left(T_{0}, T_{1}, T_{2}, \cdots\right) \mid \vDash \mathscr{T}\left(T_{0}, T_{1}, T_{2}, \ldots\right) \tau(F)\right\}=$ $\tau\left(\left\{\left(T_{0}, T_{1}, T_{2}, \cdots\right) \mid E_{\operatorname{Tr}\left(T_{0}, T_{1}, T_{\cdots}, \ldots\right)}\right\}\right)$, for each arithmetical function $\tau$.

Lemma 11 is Feferman's transformation lemma [3] with "forcing" replaced by "truth" and "generic sequence" replaced by "arbitrary sequence."

Lemma 12. Let $\mathfrak{F}$ be a sentence of $\mathfrak{L}^{*}$ whose only $\mathfrak{\Im}_{i}$-symbols are $J_{0}, J_{1}, \cdots, \Im_{n}(n \geqq 0)$. Let $T_{0}, T_{1}, \cdots, T_{n}$ be an arbitrary finite sequence of sets of natural numbers. Then the conditional probability that $\mathcal{F}$ is true, given that $\Im_{i}=T_{i}$ for all $i \leqq n$, is either 0 or 1 .

Let $X^{\omega}$ be a variable of $\mathcal{L}^{*}$ restricted to sets of natural numbers in $\operatorname{Mr}\left(T_{0}, T_{1}, T_{2}, \cdots\right)$, and let $B\left(X^{\omega}\right)$ be a formula of $\mathscr{L}^{*}$ with all quantifiers bounded. For each formula $B\left(X^{\omega}\right)$ and each sequence $T=\left(T_{0}, T_{1}, T_{2}, \ldots\right)$, we define the absolute measure of $\hat{X}^{\omega} B\left(X^{\omega}\right)$, denoted by $\mu_{a}^{T}\left(\hat{X}^{\omega} B\left(X^{\omega}\right)\right)$, as follows: let $J_{B}$ be some $\Im_{i}$-symbol not occurring in $B\left(X^{\omega}\right)$, then $\mu_{a}^{T}\left(\hat{X}^{\omega} B\left(X^{\omega}\right)\right)$ is the conditional probability that $B\left(\mathcal{J}_{B}\right)$ is true given that $J_{i}=T_{i}$ for every $\Im_{i}$-symbol occurring in $B\left(X^{\omega}\right)$. We say $\operatorname{Tr}\left(T_{0}, T_{1}, T_{2}, \cdots\right)$ is measure-complete if for every pair of formulas $B_{0}\left(X^{\omega}\right)$ and $B_{1}\left(X^{\omega}\right)$, if $\hat{X}^{\omega} B_{0}\left(X^{\omega}\right)=\hat{X}^{\omega} B_{1}\left(X^{\omega}\right)$ is true in $\operatorname{Tr}\left(T_{0}, T_{1}, T_{2}, \cdots\right)$, then $\mu_{a}^{T}\left(\hat{X}^{\omega} B\left(X^{\omega}\right)\right)=\mu_{a}^{T}\left(\hat{X}^{\omega} B\left(X^{\omega}\right)\right)$. Thus, in a measure-complete model, we can associate absolute measure with sets rather than definitions of sets. 
Lemma 13. With probability one, $\operatorname{Tr}\left(T_{0}, T_{1}, T_{2}, \cdots\right)$ is a measurecomplete model of $Z F$ in which the absolute measure is definable.

Solovay [9] has results virtually identical with Theorem 10 and Lemma 12 , save that $T_{0}, T_{1}, T_{2}, \cdots$ is always a sequence generic in his sense.

Lemma 14. With probability one, $\mathfrak{T l}\left(T_{0}, T_{1}, T_{2}, \cdots\right)$ is a measurecomplete model of $Z F$, then the absolute measure agrees with Lebesgue measure on all Lebesgue-measurable sets, is translation invariant, and is countably additive.

Lemma 15. With probability one, the countable axiom of choice holds in $\operatorname{Tr}\left(T_{0}, T_{1}, T_{2}, \cdots\right)$.

The result of Solovay [9] we quoted on the first page of this paper follows from Lemmas 13 through 15 . The translation invariance follows from some hasic facts about random variables. The countable additivity of the absolute measure follows from the measure-completeness and the countable additivity of Borel measure. In short, the sets of reals in a countable, measure-complete model form a good copy of a certain countable family of Borel sets in the "real" world. We wish to stress that this last fact has no vital connection with the ideas of forcing and genericity.

Let $\mathcal{F}$ be a sentence of $Z F$. Then the Borel set $\left\{T \mid F_{\mathscr{N}(T)} F\right\}$ has measure 0 or 1 by the $0-1$ law. $^{2}$ Let $F E T_{1}(\mathscr{I T})$, (the fundamental equivalence type of a one-element extension of $\mathfrak{T})$, consist of all $F$ such that $\left\{T \mid F_{\mathscr{T}(T)} \mathfrak{F}\right\}$ has measure 1 ; as we saw above, $F E T_{1}(\mathfrak{T})$ includes $Z F+V \neq L$. All Cohen-generic extensions $\mathfrak{T}(T)$ have the same elementary equivalence type, and that type is not $F E T_{1}(\mathfrak{T K})$. In [6] we will define the fundamental equivalence types of general, countable models.

Call $T$ fundamental if $\operatorname{ml}(T)$ has the fundamental equivalence type. Several persons have observed: a set is fundamental if and only if it has the same degree of nonconstructibility as some Solovay-generic set. When we pass from sets to degrees, we pass from forcing to measure-theoretic uniformity. In [7] Scott and Solovay provide an interesting general theory which makes possible an accurate comparison between the forcing approach and the measure-theoretic uniformity approach.

2 Professor Simon Kochen suggested the use of the 0-1 law here in place of Lemma 12. 


\section{REFERENCES}

1. P. J. Cohen, The independence of the continuum hypothesis. I, Proc. Nat. Acad. Sci. U.S.A. 50 (1963), 1143-1148.

2. The independenie of the continuum hypothesis. II, Proc. Nat. Acad. Sci. U.S.A. 51 (1964), 105-110.

3. S. Feferman, Some applications of the notions of forcing and generic sets, Fund. Math. 56 (1965), 325-345.

4. G. Kreisel, The axiom of choice and the class of hyperarithmetic functions, Indag. Math. 24 (1962), 307-319.

5. G. E. Sacks, Measure-theoretic uniformity in recursion theory, hyperarithmitic analysis, and set theory, in preparation.

6. - On the fundamental equivalence type of a countable model, in preparation.

7. D. Scott and R. Solovay, Boolean-valued models and forcing, (to appear).

8. R. Solovay, The measure problem, Abstract 65T-62, Notices Amer. Math. Soc. 12 (1965), 217.

9. — The measure problem, (to appear).

10. C. Spector, Measure-theoretic construction of incomparable hyperdegrees, J. Symbolic Logic 23 (1958), 280-288.

\section{Cornell University}

